\title{
Covid-19: avaliação comportamental de moradores das zonas rural e urbana usuários do SUS, no âmbito da atenção primária, do munícipio de Cláudio - Minas Gerais - Brasil
}

\author{
Tácio Gonçalves Nogueira Fonseca ${ }^{1}$ \\ Elisângela Pinto dos Santos \\ Cleonice Ferreira Rabelo ${ }^{1}$ \\ Melissa Amaral Pacheco ${ }^{1}$ \\ Amanda Gonçalves Franco ${ }^{2}$ \\ Geraldo Alberto Pinheiro de Carvalho' \\ Sérgio Candido Dias ${ }^{1}$ \\ Elimário Venturin Ramos ${ }^{1}$ \\ Aline Batista Gonçalves Franco ${ }^{1}$
}

A organização mundial da saúde (OMS) e os governos de todo o mundo enfrentam hoje uma grave crise, a pandemia pelo novo Coronavírus. Segundo a OMS, a doença foi identificada pela primeira vez em Wuhan, na China no final de 2019. O vírus é causador de uma síndrome respiratória grave que tem gerado mortes em todo o mundo. De acordo com Zhu et al. [1], o SARSCov-2 como é denominado o novo Coronavírus, provém de um grupo de sete Coronavírus com capacidade de agredir seres humanos, dentre os quais cita-se o 229E, OC43, HKU1,NL63,MERS-CoV e o SARS - CoV. A denominação Covid-19 foi designada pela OMS e se refere à doença por Coronavírus descoberta no final de 2019.

A transmissão ocorre através de gotículas produzidas pelos indivíduos que, ao espirrar ou tossir, contaminam o ambiente em geral. O contágio também pode acontecer de maneira indireta, quando o indivíduo toca em superfícies contaminadas levando posteriormente as mãos no nariz e nos olhos. Além disso, alguns pesquisadores pontuam que as gotículas produzidas pelos indivíduos ao falar e respirar podem percorrer metros de distância e possuir potencial de contágio, devendo a via de transmissão pelo ar ser considerada também como um meio importante de transmissão [2].

Segundo a OMS [3], o tempo entre a exposição ao vírus e o início dos sintomas (período de incubação) pode variar de 2 a 14 dias, sendo a tosse, febre e dificuldade respiratória os principais sintomas da doença. Com relação ao contágio, muitos estudos relatam a possibilidade de transmissão do novo Coronavírus mesmo por pessoas assintomáticas. Segundo Zou et al. [4], o contágio pode ocorrer de 1 a 2 dias antes de surgirem os sintomas a depender da viremia presente. No trabalho de Li et al. [5], foi possível perceber que a maioria das contaminações em Wuhan na China, ocorreram por pessoas sem sintomas ou com sintomas leves. Anderson [6], também relata a possibilidade de transmissão do SARS COV-2 antes da manifestação dos sintomas pelos indivíduos, o que o diferencia do SARS- Cov originado na China no ano de 2002.

Entre as medidas de prevenção mais preconizadas pelas organizações mundiais e sistemas de saúde estão a lavagem frequente das mãos, uso de máscaras de proteção e o distanciamento entre pessoas. Além disso, segundo

${ }^{1}$ Faculdade São Leopoldo Mandic, Curso de Odontologia. Rua José Rocha Junqueira, 13, Swift, 13045-755, Campinas, SP, Brasil Correspondência para /
Correspondence to: AG FRANCO. E-mail: aalinebgfranco@yahoo.com.

${ }^{2}$ Universidade de Itaúna, Faculdade de Odontologia. Itaúna, MG, Brasil.

$\square \square \square \square$

Como citar este artigo / How to cite this article

Fonseca TGN, dos Santos EP, Rabelo CF, Pacheco MA, Franco AG, de Carvalho GAP, Dias SC, Ramos EV, Franco ABG. A. Covid-19: avaliação comportamental de moradores das zonas rural e urbana usuários do SUS, no âmbito da atenção primária, do munícipio de Cláudio - Minas Gerais - Brasil. InterAm J Med Health 2020;3:e202003046. 
segundo Anderson [6] a responsabilidade de se existem diferenças importantes nos posicionamentos

cada pessoa também representa importante papel no dos moradores das zonas rural e urbana. cumprimento de tais ações.

Para o enfrentamento à Covid-19, a grande extensão territorial do Brasil, com a variedade de costumes hábitos dos indivíduos, juntamente com a diversificação das assistências à saúde compatíveis com cada localidade, representam um desafio para as autoridades na questão de planejamento de ações. Neste contexto, o governo federal oferece a assistência à saúde através do sistema único de saúde (SUS), tendo como porta de entrada dos usuários o Programa de Saúde da Família (PSF), no qua a abordagem vai além do tratamento de doenças, mas também a recuperação da saúde e prevenção de agravos e tem como foco a família e a comunidade, considerando aspectos ambientais, socioeconômicos e culturais.

Em 2006 o PSF deixou de ser programa e passou a ser uma estratégia permanente na atenção primária em saúde, com a justificativa de que programa possui um tempo determinado e estratégia é permanente contínua. Desse modo passou a ser denominado de Estratégia Saúde da Família - ESF como consta na Portaria de Consolidação $n^{\circ} 2$ de 28 de setembro de 2017 no seu anexo I do anexo XXII que trata dos tipos de equipes de Atenção Básica [7]

No combate à Covid-19, a abordagem regionalizada tem sido o foco de ação de muitos governos em todo o mundo, sendo que no Brasil a estrutura do SUS apresentase como um fator facilitador no enfrentamento ao novo Coronavírus, através da estratégia de saúde da família (ESF) e suas ações voltadas para a comunidade. Para que essa estratégia seja efetiva a atitude de cada pessoa se torna importante, além da dedicação da gestão e dos agentes de saúde na prestação da assistência. Abib [8], em sua obra "Teoria moral de Skinner e desenvolvimento humano", relata a importância de os indivíduos, nas suas relações sociais, se comportarem objetivando seus bens e os de seu próximo. Logo, a responsabilidade de cada pessoa no cumprimento e acato às recomendações de saúde podem contribuir para manutenção da saúde da comunidade e consequentemente estabilização da pandemia.

Portanto, o presente estudo tem como objetivo avaliar a pandemia da Covid-19 no contexto de vida dos usuários das UBSs do munícipio de Cláudio-Minas Gerais, interesse do usuário acerca da pandemia e o comportamento da população dependente desse sistem frente ao atual problema. Objetiva-se também identificar

\section{Materiais e métodos}

O presente estudo foi realizado com os usuários do sistema se saúde pública de Cláudio, município localizado Segundo a estimativa do IBGE de 2019, sua população era de 28.617 habitantes, possuindo a cidade uma cobertura da ESF (Estratégia de Saúde da Família) de $86,00 \%$, totalizando 07 equipes de saúde da familia. A assistêncià Saude da Famlia) de $86,00 \%$, sendo que deste conta com 205 profissionas ao todo, pendo que deste total 100 compoem a ESF. A população pode contar também com uma equipe multiprofissional na atenção básica, anteriormente nomeada como Núcleo ampliado de Saúde da Família (NASF-AB), um pronto atendimento, um centro de especialidades médicas, um núcleo de fisioterapia, uma farmácia e um centro de atenção psicossocial (CAPS), além de ambulatório de psicologia, todos no âmbito do SUS

O município possui em média 15 comunidades rurais. Algumas delas se encontram distantes da zona urbana o que demonstra a necessidade de ações assistenciais especializadas por parte da gestão. Para tal, o município disponibiliza motoristas que se deslocam com as equipes de saúde da família semanalmente e motocicletas que s̃o usadas pelos ACSs (Agentes comunitários de saúde) tentas localidades rurais diariamente, facilitando o acesso à saúde pela população local. Das sete ESFs, 03 atendem somente a comunidades urbanas e 04 atendem ambas as comunidades, contando essas com pontos de apoio nas comunidades com maior número de habitantes.

Diante da pandemia e seguindo o Protocolo de Manejo Clínico da COVID-19 na Atenção Primária à Saúde - versão 9, houve algumas mudanças na rotina de trabalho nas unidades de saúde. Dentre elas, a realização de visitas domiciliares pelos agentes comunitários de saúde apenas no peri-domicílio, suspensão de consultas e atendimentos odontológicos eletivos, além da incorporação da telemedicina conforme regulamenta a Portaria 467 de 20 de março de 2020

Logo, para realização do presente estudo foram abordados 164 usuários do sistema público de saúde

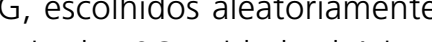
dentre as areas de abrangência das de saúde que representam 07 ESF na região oeste do estado de Minas Gerais, Brasil. segundo Anderson [6] a responsabilidade de cada pessoa também representa importante papel no cumprimento de tais ações.

Para o enfrentamento à Covid-19, a grande extensão territorial do Brasil, com a variedade de costumes e hábitos dos indivíduos, juntamente com a diversificação das assistências à saúde compatíveis com cada localidade, representam um desafio para as autoridades na questão de planejamento de ações. Neste contexto, o governo federal oferece a assistência à saúde através do sistema único de saúde (SUS), tendo como porta de entrada dos usuátios a Programa de Saúde da Família (PSF), no qual a abordagem vai além do tratamento de doenças, mas também a recuperação da saúde e prevenção de agravos e tem como foco a família e a comunidade, considerand
aspectos ambientais, socioeconômicos e culturais.

Em 2006 o PSF deixou de ser programa e passou a ser uma estratégia permanente na atenção primária em saúde, com a justificativa de que programa possu um tempo determinado e estratégia é permanente $e$ contínua. Desse modo passou a ser denominado de Estratégia Saúde da Família - ESF como consta na Portaria de Consolidação $n^{\circ} 2$ de 28 de setembro de 2017 no seu anexo I do anexo XXII que trata dos tipos de equipes de Atenção Básica [7]

No combate à Covid-19, a abordagem regionalizada tem sido o foco de ação de muitos governos em todo o mundo, sendo que no Brasil a estrutura do SUS apresentase como um fator facilitador no enfrentamento ao novo se como um fator faclitador no enfrentamento ao novo CoSF) e suas açós vola estrategia de saúde da família (ESF) e suas ações voltadas para a comunidade. Para que essa estratégia seja efetiva a atitude de cada pessoa se torna importante, além da dedicação da gestão e dos agentes de saúde na prestação da assistência. Abib [8], em sua obra "Teoria moral de Skinner e desenvolvimento humano", relata a importância de os indivíduos, nas suas relações sociais, se comportarem objetivando seus bens e os de seu próximo. Logo, a responsabilidade de cada pessoa no cumprimento e acato às recomendaçōes de saúde podem contribuir para manutenção da saúde da comunidade e consequentemente estabilização da pandemia.

Portanto, o presente estudo tem como objetivo avaliar a pandemia da Covid-19 no contexto de vida dos usuários das UBSs do munícipio de Cláudio-Minas Gerais, analisando o interesse do usua dio acládio-Minas Gera frente ao atual problema. Objetiva-se também identificar se existem diferenças importantes nos posicionamentos dos moradores das zonas rural e urbana.

\section{Materiais e métodos}

presente estudo foi realizado com os usuários do sistema se saúde pública de Cláudio, município localizado na região oeste do estado de Minas Gerais, Brasil. segundo a estimativa do IBGE de 2019, sua população era de 28.617 habitantes, possuindo a cidade uma cobertura da ESF (Estratégia de Saúde da Família) de $86,00 \%$, 205 profissionis ao todo . sendo que deste total 100 compoem a ESF. A população pode contar também com uma equipe multiprofissional na atenção básica, anteriormente nomeada como Núcleo ampliado de Saúde da Família (NASF-AB), um pronto atendimento, um centro de especialidades médicas, um núcleo de fisioterapia, uma farmácia e um centro de atenção psicossocial (CAPS), além de ambulatório de psicologia, todos no âmbito do SUS.

O município possui em média 15 comunidades rurais. Algumas delas se encontram distantes da zona urbana - que demonstra a necessidade de ações assistenciais especializadas por parte da gestão. Para tal, o município disponibiliza motoristas que se deslocam com as equipes de saúde da família semanalmente e motocicletas que são usadas pelos ACSs (Agentes comunitários de saúde) técnicos de enfermagem destas localidades rurais diariamente, facilitando o acesso à saúde pela população local. Das sete ESFs, 03 atendem somente a comunidades urbanas e 04 atendem ambas as comunidades, contando essas com pontos de apoio nas comunidades com maior número de habitantes.

Diante da pandemia e seguindo o Protocolo de Manejo Clínico da COVID-19 na Atenção Primária à Saúde - versão 9 , houve algumas mudanças na rotina de trabalho nas unidades de saúde. Dentre elas, a realização de visitas domiciliares pelos agentes comunitários de saúde apenas no peri-domicílio, suspensão de consultas e atendimentos odontológicos eletivos, além da incorporação da telemedicina conforme regulamenta a Portaria 467 de 20 de março de 2020

Logo, para realização do presente estudo foram abordados 164 usuários do sistema público de saúde dentre as áreas de abrangência das 06 unidades básicas de saúde que representam $07 \mathrm{ESF}$ 
Os usuários foram devidos em 2 grupos: Grupo A - 90 moradores da zona urban Grupo B - 74 moradores da zona rura

Canta participante recebeu um questionário (ANEXO 1), totalizando 164 questionários com 22 perguntas cada todos de mesmo conteúdo e com a mesma quantidade de perguntas, relacionadas às práticas de higiene e condutas sociais frente a pandemia atual sendo tais documentos produzidos e financiados pelos próprios pesquisadores.

As abordagens foram realizadas pelos pesquisadores e também pelos colaboradores da pesquisa, instruídos e tamben pelos colaboradores da pesquisa, instruidos sobre a pratica e segundo todos os protocolos de cuidados preconizados pelo ministério da saúde foi observado com o devido rigor. As questões foram arguidas pelos pesquisadores e colaboradores não havendo assim transição de canetas entre entrevistados e questionadores.

\section{Discussão}

Fatores como acesso à educação, lazer, cultura, e não exposição a agentes nocivos dentre outros, influenciam na saúde dos indivíduos, sendo a qualidade de vida um fator importante na manutenção da saúde. Logo podemos perceber que a ideia principal da ESF (Estratégia de Saúde da Família) se baseia em considerar - processo saúde-doença como multifatorial, exigindo a melhor abordagem ao doente.

Em tempos de pandemia, o cuidado com a própria saúde, a prática dos hábitos de higiene e o respeito às recomendações dadas pelos governantes representam condutas compatíveis com a responsabilidade socia de cada pessoa, resguardando a saúde do indivíduo e consequentemente da comunidade que se apresenta à sua volta. Diante disso, as equipes de saúde da família do suS (Sistema Único de Saúde), por serem conhecedoras da comunidade local, adquirem a capacidade de entender avaliar a conduta dos indivíduos frente a atual pandemia. A função realizada pelos ACS (Agentes Comunitários de Saúde) se mostra muito importante justamente pelo fato de atuarem junto das pessoas, pois reportam à toda a equipe de saúde características comuns, como costumes e condutas diárias da população local.

No contexto da comunidade rural, a atuação dos ACS apresenta certas particularidades em vista das funções realizadas pelos mesmos no ambiente urbano. Apesar dos esforços e de toda a estrutura disponibilizada pela gestão pública, alguns problemas ainda são enfrentados por tais profissionais. As distâncias elevadas a serem percorridas bem como a assistência a localidades ribeirinhas de difícil acesso, principalmente no período das chuvas, dificultam a missão dos ACS na assistência à população e nas campanhas de conscientização. No entanto, mesmo com as presentes restrições, as visitas e a assistência aos moradores são realizadas pelas equipes de saúde da família com efetividade.

A responsabilidade social de cada indivíduo em cuidar da sua saúde e consequentemente resguardar a saúde das demais pessoas foi abordada por Anderson [6], em estudo realizado no Imperial College de Londres. Segundo ele, o comportamento e a conscientização de cada indivíduo possuem fundamental importância no controle da pandemia podendo ser tão relevantes quanto as ações governamentais de enfrentamento à pandemia.

No presente trabalho, praticamente todos os usuários entrevistados pertencentes aos dois grupos conhecem ou já ouviram falar da pandemia do novo Coronavírus, sendo que apenas 2 moradores ao todo, ambos oriundos do grupo B (zona rural), nunca ouviram falar da Covid-19. A maior parte das pessoas, no grupo A, tomou conhecimento da pandemia através da televisão e das redes sociais. Já os indivíduos do grupo B (zona rural),

Uma parcela considerável dos entrevistados da zona rural também relatou ter acesso a tal informação através de amigos, parentes e agentes comunitários de saúde o que demonstra a diferença das realidades entre os dois grupos de moradores. O número de pessoas que tomou conhecimento da pandemia através dos ACS foi praticamente igual nos dois grupos

Neste contexto, apesar de alguns moradores não terem acesso a aparelhos de TV e principalmente à internet, a informação e o conhecimento permeiam a comunidade, observando-se a importante função realizada pelos ACS e pelos profissionais das Unidades básicas de saúde em levar o conhecimento e a conscientização à população local. Logo, podemos perceber a grande restrição de acesso à internet por parte dos moradores do campo e a importância do rádio na disseminação da informação principalmente nos ambientes mais distantes.

Diante dos expostos acima, podemos ressaltar que a restriça de acesso à informação representa um problema para o enfrentamento da crise pela população, pois educacionais oferecidas pelo governo. A observação de pessoas residentes na zona rural que ainda desconhecem a existência da pandemia mesmo com todas as ações presentes, representa um dado importante e nos remete a ideia de um certo "isolamento social permanente" a que se submetem certos grupos de pessoas na atualidade. Em outra abordagem, a ideia de que o Coronavírus pode levar a morte é amplamente conhecida por praticamente todos os usuários abordados. Com relação ao contágio, o fato de que certas pessoas, mesmo sem sinais da doenca podem estar contaminadas, representa a op dois grupos. Diante disso, a malor parte das dois grupos. Diante disso, a maior parte das pessoas no contexto urbano e rural concordam com o fato de que um indivíduo, mesmo sem nenhum sinal de gripe ou tosse, pode estar contaminada e também transmitir o vírus. A transmissibilidade por pessoas sem sintomas ou com sintomas leves representa o foco de muitas pesquisas atuais. Hu et al. ( 2020 ) relatou em seu estudo realizado na China, a existência de pessoas portadoras do novo Coronavírus e que se encontravam totalmente assintomáticos. Segundo Li et al. [5] a transmissão por pacientes assintomáticos ou pré sintomáticos pode ocorrer, podendo o período infeccioso perdurar por 10 dias ou mais após a manifestação dos sintomas [4]. No entanto, as incertezas sobre a transmissibilidade da Covid-19 ainda existem e a comunidade científica busca mais informaçōes sobre o comportamento do vírus.

Neste contexto, a transmissibilidade elevada do novo Coronavírus exige por parte da comunidade e dos governantes, medidas importantes para auxílio na contenção da doença, uma vez que as abordagens farmacológicas ainda estão em desenvolvimento. A lavagem das mãos, o distanciamento social, o arejamento de ambientes fechados além da limpeza de objetos, superfícies e restrição ou proibição ao funcionamento de escolas, dentre outros, são condutas importantes citadas como "intervenções não farmacológicas (INF) " no estudo de Garcia \& Duarte [9].

Um dado importante é que, apesar de o distanciamento entre as pessoas, explicado aos usuários como "manter distância segura das pessoas, amigos e até parentes" no combate à COVID-19, possuir importância para a maior parte dos entrevistados nos dois grupos, uma minoria destes indivíduos demonstrou não considerar relevante tal conduta, não a colocando em prática.

Com relação aos sintomas da Covid-19, a maioria dos participantes nos dois grupos relatou conhecê-los. Porém, no grupo B, apesar de não representarem a maioria, algumas pessoas desconhecem as manifestações físicas a doença. 0 entendimento dos principais sintomas, aem como das orientações dadas pelos órgãos de saúde se torna fundamental, uma vez que ao apresentar qualquer alteração suspeita, o usuário poderá decidir pela procura dos serviços de saúde ou não. A opção pelo auto isolamento e consulta médica remota, caso esta esteja disponível e desde que não haja manifestação de sintomas graves, diminui a circulação de pessoas nas ruas, representando a colaboração dos indivíduos com a comunidade e com o sistema público de saúde [10].

A redução na circulação de pessoas nos ambientes de assistência à saúde foi abordada por Nacoti et al. [11] como um dos benefícios dos atendimentos remotos. Logo, o acesso à informação juntamente com a responsabilidade social contribui para uma diminuição da circulação de usuários dentro das unidades e também nas ruas, com respeito às exigências de distanciamento social e boas práticas de higiene pessoal.

Com relação aos questionamentos sobre as condutas de higiene, a maioria dos entrevistados dos dois grupos relatou melhora dos hábitos pessoais de higiene com o advento da pandemia, afirmando realizar a limpeza das mãos a todo momento e também dos alimentos que compram, antes de armazená-los em casa. Foi importante verificar que no ambiente do campo, uma quantidade relevante de usuarios (não representantes da maioria) possuem 0 hábito de lavar as mãos somente antes das refeicões, não higienizando as mãos ao chegar e sair de casa e nem tomando cuidados quanto a desinfecção dos alimentos que compram. Portanto, apesar do entendimento sobre as novas práticas de higiene e cuidados preconizados pelo governo se encontrar presente na realidade da maioria dos usuários. Uma certa deficiência na aplicação dessas ações pôde ser notada no contexto rural.

Com relação a utilização do álcool um achado relevante foi encontrado. Segundo as recomendações da OMS [3], lavar as mãos apenas com álcool sem remover toda as sujidades das mãos com água e sabão previamente, nâo representa medida eficaz. A maioria dos entrevistados lavam as mãos previamente com água e sabão antes de higienizar com álcool 70\% e demonstram conhecimento sobre qual álcool utilizar. No entanto algumas pessoas, principalmente da zona rural, desconhecem a indicação do álcool 70\%, relatando utilizar qualquer álcool. Observa-se neste contexto a necessidade de um maior 
interesse e busca por informação por parte das pessoas além da necessidade de campanhas educacionais mais específicas sobre detalhes de higienização e produtos a serem utilizados. Lavar as mãos regularmente e usar máscaras de proteção podem evitar a transmissão do COVID -19 sendo também essas, as medidas apontadas como mais eficientes pela maior parte das pessoas.

Outro achado relevante observado na pesquisa, indica que a maior parte das pessoas já foram orientadas ou informadas sobre o correto manuseio das máscaras de proteção e por qual motivo usá-las A utilização das máscaras se torna importante, mas desd mascaras se torna importante mas desce que usadas corretamente e de acordo com as preconizaçoes dos órgáos de saúde, como relata a OMS [3]. No entanto, uma minoria dos entrevistados alegou não ser importante seu uso contínuo, comparecendo em público sem máscaras. O uso das máscaras de proteção representa importante medida no controle da pandemia e é alvo de discordâncias na comunidade científica. De acordo com Feng $S$ et al. [12] a sua utilização possui potencia para redução da contaminação comunitária e deveria ser adotada por todas as pessoas, com ou sem sintomas da doença. No entanto, segundo a OMS [3], a indicação do uso das máscaras pode acarretar no relaxamento de algumas medidas importantes pela possibilidade de extrapolar a ideia de proteção, colocando em risco a saúde das pessoas, sendo indicado por indivíduos portadores do vírus, suspeitos de contágio e também seus cuidadores. Além disso, esta organização ressalta sobre a importância do uso correto da mesma.

Ainda com relação ao uso das máscaras de proteção metade dos entrevistados alegou substituí-las todos os dias. No entanto, apesar da maioria dos entrevistados lavar as máscaras diariamente e utilizarem uma máscara por dia, uma boa parcela dos entrevistados usa a mesma proteção por até dois dias Observou-se também que uma pequena quantidade dos usuários afirmou utilizar a máscaras de proteção por até cinco dias. A utilização prolongada das máscaras ocasiona na perda da sua efetividade, permitindo o aumento da susceptibilidade à contaminação por microrganismos. Logo, as máscara são relevantes no controle da emissão de gotículas produzidas por espirros, tosses e também pelo aerosso oriundo da respiração e da fala dos indivíduos. Existem comprovações do início do século passado que a fala a respiração podem gerar partículas de aerossol como observado por Duguid [13]. Segundo van Doremalen et al. [14] o SARS-CoV-2 pode permanece em aerossol no a possuindo uma meia-vida da ordem de uma hora neste ambiente podendo ser possível a sua transmissibilidade . de transmissão por pacientes assintomáticos e a chance de contaminação por aerossóis, o uso das máscaras de proteção se mostra fundamental, principalmente até que estudos mais conclusos forneçam amparo para decisões contrárias.

Quando questionados sobre acesso às redes sociais, a maioria das pessoas não acredita em toda informação que recebe se preocupando em confirmar a veracidade das notícias que recebem ante de compartha tas. No Un numo respor possuir acesso à internet, demonstranto a lim por náo à internet, demonstrando a limitaçáo de

A internet, através das redes sociais pode representar uma importante ferramenta de complementação das ações de conscientização realizadas pelos ACS e pelas equipes de saúde da família pois permitem mais acesso às recomendações pela comunidade local. Todavia, vantagens como acessibilidade e a velocidade de disseminação de informações podem acarretar em problemas como o risco de compartilhamento de notícias falsas.

De acordo com a OMS [3] uma "infodemia maciça" de notícias verdadeiras e falsas tem ocorrido com o grande aumento nas informações e notícias relatadas sobre o vírus o que dificulta o acesso às recomendacõos confiáveis. Logo, podemos observar a preocupação dessa entidade com a ocorrência de notícias falsas permeando as informações verídicas, provavelmente por acarretarem em prejuízo das ações preconizadas pelo poder público e consequentemente atrasos na estabilização e contenção da crise

Diante dos achados acima percebe-se que as campanhas do governo federal e o trabalho das equipes de saúde da familia possuem efeitos na comunidade, revelando que a maioria dos moradores possuem conhecimentos coerentes relativos ao novo Coronavírus, sua prevenção, transmissibilidade e riscos. Particularmente na zona rural, observou-se um número maior de pessoas carentes de informação, felizmente não representando a maioria deles.

O comportamento de cada pessoa juntamente com a presença contínua dos profissionais dentro das UBS, a dedicação de médicos, enfermeiros, dentistas e de todos os profissionais ali presentes, além dos esforços da gestão para a continuidade da assistência com o fornecimento de informações, equipamentos de proteção individua e manutenção dos atendimentos, possuem importante

\section{Conclusão}

O conhecimento sobre a pandemia e seus riscos apresenta-se difundido por toda a comunidade urbana $e$ rural. No entanto a aplicação das medidas de segurança contra a Covid-19 passa pela responsabilidade social e também pela educação inerente a cada pessoa. Logo necessidade de um maior engajamento interessena busca porinformações e dec por informa cos ededicação por parte dos moradores fo percebida em muitos questionamentos, representando um papel fundamental no combate à doença.

Todos os esforços do governo e dos agentes de saúde dependem também da responsabilidade e do comprometimento de cada indivíduo para a conquista do objetivo de controle da pandemia. Portanto, a prestação da assistência à saúde atrelada ao comprometimento de toda a comunidade local e consequentemente de toda a sociedade pode gerar benefícios no enfrentamento do novo Coronavírus.

\section{REFERÊNCIAS}

1. Zhu N, Zhang D, Wang W, et al. A Novel Coronavirus from Patients with Pneumonia in China, 2019. N Engl J Med. 2020 feb[cited 2020 june 23];382(8):727733. Available from: doi:10.1056/NEJMoa2001017

2. Morawska L, Cao J. Airborne transmission of SARSCoV-2: The world should face the reality. Environ Int 2020 apr [cited 2020 june 23]; 139:105730. Available from: doi: 10.1016/jenvint.2020.105730

3. Organização Mundial da Saúde. (2020). Novo Coronavírus (2019-nCoV): relatório da situação, 13. Organização Mundial da Saúde. https://apps.who.int/iris/handle/10665/330778

4. Zou L, Ruan F, Huang $M$, Liang L, Huang $H$, Hong $Z$ et al. SARS-COV-2 viral load in upper respiratory Z, ecimens of inferted patients. N Engl J Med 2020 specimens of infected patients. N Engl J Med. 2020 $\begin{array}{lccc}\text { marlcited } & 2020 & \text { june 23];382(12):1177-1179, } \\ \text { Available } & \text { from: } & \text { https://doi:10.1056/NEJMc2001737 }\end{array}$
5. Li R, S Pei, B Chen, Y Song, T Zhang, W Yang, J Li R, Pei S, Chen $B$, et al. Substantial undocumented infection facilitates the rapid dissemination of novel coronavirus (SARS-COV-2). Science. 2020 malcited 2020 june181365(6490) 489493. Available from: 10.1126/science. abb3221.

6. Anderson RM, Heesterbeek $H$, Klinkenberg $D$, Hollingsworth TD. How will country-based mitigation measures influence the course of the COVID-19epidemic? Lancet.2020 mar[cited 2020 june 18];395(10228):931934. Available from:10.1016/50140-6736(20)30567-5

7. Ministério da Saúde. Secretaria de Atenção Primária à Saúde - Protocolo de Manejo Clínico do COVID-19 na Atenção Primária à Saúde. (Versão 5). Brasilia - DF, março 2020. 8. Abib, JAD. Teoria moral de Skinner e o desenvolvimento humano. Psicol. Reflexo. Crit. 2001[cited 2020 june 23];14(1):107-117.Available from: <http// www. scielo.br/scielo. . php? script=sci arttext\&pid=\$0102$79722001000100009 \& \operatorname{lng}=$ en\&nrm=iso $>$. ISSN 1678-7153. http.//dx.doi.org/10.1590/S0102-79722001000100009. 9. Garcia LP, Duarte E. Intervenções não farmacológicas para o enfrentamento à epidemia da Covid-19 no Brasil. Epidemiol Serv Saúde 2020 mai [citado 2020 june 23];29(2): [cerca de 4p]. Disponível em: https://doi.org/10.5123/s1679-49742020000200009.

10. UK Government, Department of Health and Social Care Coronavirus action plan.2020 mar 3https://www. gov.uk/government/publications/coronavirus-action-plan.

11. Nacoti M, Ciocca A, Giupponi A, Brambillasca P, ussana F, Pisano M, et al. At the epicenter of the covid-19 pandemicandhumanitariancrisesinltaly:changingperspectives on preparation and mitigation. New England Journal of Medicine. 2020 mar [cited 2020 june 18][about 5p.] Available from: https://catalyst.nejm.org/doi/full/10.1056/CAT.20.0080

12. Feng $S$, Shen $C$, Xia $N$, Song $W$, Fan $M$, Cowling BJ. Rational use of face masks in the COVID-19 pandemic. Lancet Respir Med .2020 May [cited 2020 june 23];8(5):434-436. Available from: https://doi.org/10.1016/\$2213-2600(20)30134-X

13. Duguid JP. The size and the duration of aircarriage of respiratory droplets and droplet-nuclei. J Hyg 
14. Van DN, Bushmaker T, Morris DH, et al. Aerosol and Surface Stability of SARS-CoV-2 as Compared with SARS-CoV-1. N Engl J Med. 2020 apr[cited 2020 june 23];382(16):1564-1567. Https://doi:10.1056/NEJMc2004973

15. Giovanella L. A atenção primária à saúde nos países da União Européia: configurações e reformas organizacionais na década de 1990. Cad. Saúde Pública .2006 May [cited 2020 June 23]; 22(5): 951-963. Available from: http://dx.doi.org/10.1590/S0102311X2006000500008.

16. Conill Eleonor Minho. Ensaio histórico-conceitual sobre a Atenção Primária à Saúde: desafios para a organização de serviços básicos e da Estratégia Saúde da Família em centros urbanos no Brasil. Cad. Saúde Pública .2008 [cited 2020 June 23]; 24(Suppl 1): s7-s16. Available from: http://dx.doi.org/10.1590/S0102-311X2008001300002.

17. Werneck GL, Carvalho MS. A pandemia de COVID-19 no Brasil: crônica de uma crise sanitária anunciada. Cad. Saúde Pública.2020 may [cited 2020 June 18] ;36(5): e00068820. Availablefrom:http://www.scielo.br/scielo.php? Script=sci_ arttext\&pid=S0102-311X2020000500101\&lng=en. Epub May 08,2020. https://doi.org/10.1590/0102-311×00068820.

18. Chen N, Zhou M; Dong $X, Q u$ J, Gon, $F$ Han $Y$, et al. Epidemiological and clinical characteristics of 99 cases of 2019 novel coronavirus pneumonia in Wuhan, China: a descriptive study. Lancet.2020 jan [cited 2020 june 23];396(10223);507-513. Available from: https:// doi:10.1016/S0140-6736(20)30211-7.

19. Hellewell J, Abbott S, Gimma A, et al. Feasibility of controlling COVID-19 outbreaks by isolation of cases and contacts. Lancet Glob Health. 2020 feb [cited 2020 june 23]; 8(4):488ه496. Doi:10.1016/\$2214-109X (20)30074-7

20. Lloyd-Smith, J., Schreiber, S., Kopp, P. et al. Superspreading and the effect of individual variation on disease emergence. Nature.2005 nov [cited 2020 june 23];438: 355-359. Available from: https://doi.org/10.1038/nature04153

21. Prasad A, Prasad M. SARS-CoV-2: o surgimento de um patógeno viral causando estragos na existência humana. J. Genet. 2020 apr [cited 2020 june 23]; 99 (1): 37. Available from: https;//doi: 10.1007 / s12041-020-01205-x
22. Starfield, B. Atenção Primária: equilíbrio entre necessidades de saúde, serviços e tecnologia. Brasília: UNESCO, Ministério da Saúde, 2002. 726 p.

23. Brasil. Ministério da Saúde. Portaria n . 648, de 28 de março de 2006. Política nacional de atenção básica. Diário Oficial da União, Brasília (DF): 2006 mar 29; seção 1:71-75. [Série A. Normase Manuais Técnicos] [Série Pactos pela Saúde 2006, v. 4].

24. Brasil. Ministério da Saúde. Portaria n. 467, de 20 de março de 2020.Dispõe, em caráter excepcional e temporário, sobre as ações de Telemedicina, com o objetivo de regulamentar e operacionalizar as medidas de enfrentamento da emergência de saúde pública de importância internacional previstas no art. $3^{\circ}$ da Lei $n^{\circ} 13.979$, de 6 de fevereiro de 2020, decorrente da epidemia de COVID-19. Diário Oficial da União, Brasília (DF):2020 mar 23; seção1:1

25. Brasil. Ministério da Saúde. Portaria GM/MS n. 4.279, de 30 de dezembro de 2010. Estabelece diretrizes para a organização da Rede de Atenção à Saúde no âmbito do Sistema Único de Saúde (SUS). Diário Oficial da União, Braślia (DF): 2010 dez 31; seção 1;88.

26. Brasil. Ministério da Saúde. Portaria n 639, de 31 de março de 2020. Dispõe sobre a Ação Estratégica "O Brasil Conta Comigo - Profissionais da Saúde", voltada à capacitação e ao cadastramento de profissionais da área de saúde, para o enfrentamento à pandemia do coronavírus (COVID-19). Diário Oficial da União, Brasília (DF);2020 abr 2; Seção1: 76.

27. Skinner, B. F. (1971). Beyond freedom and dignity. New York: Alfred A. Knopf. 28. Skinner, B. F. (1978). Reflections on behaviorism and society. Englewood Cliffs: Prentice-Hall. 